\title{
Application of Fruit Fly Algorithm for Security Constrained Optimal Power Flow Problem
}

\author{
S. Sakthivel \\ Professor, \\ V.R.S. College of Engineering and \\ Technology, \\ Arasur-607 107, \\ Villupuram Dt, Tamil Nadu, India.
}

\author{
K. Kavipriya, P. Poovarasi \\ UG students \\ V.R.S. College of Engineering and \\ Technology, \\ Arasur-607 107, \\ Villupuram Dt, Tamil Nadu, India.
}

\author{
B. Prema \\ UG students \\ V.R.S. College of Engineering and \\ Technology, \\ Arasur-607 107, \\ Villupuram Dt, Tamil Nadu, India.
}

\begin{abstract}
Optimal power flow (OPF) is a major task in power system economics and operation. In OPF power real power outputs from the generators of a power system are so adjusted that the total production cost is minimum. Security constraint OPF (SC-OPF) is minimizing the cost keeping line flows within their respective limits for security reasons. Real power output from generators, generator bus voltage magnitudes, var outputs from shunt compensators and transformer tap settings are controlled for optimizing the total fuel cost in this OPF problem. This proposed work considers the bio inspired fruit fly algorithm (FFA) for optimally selecting the values for control variables. The proposed algorithm is simple, with less number of parameters and easy to implement. The performance of this algorithm in OPF task is tested on IEEE 30 bus test system. Numerical results are compared to literature results and found to be improved.
\end{abstract}

\section{Keywords}

Optimal power flow, security constraint optimal power flow, bio inspired algorithm, line flow limit.

\section{INTRODUCTION}

OPF control in power systems has a direct impact on system security and economic dispatch. It has become one of the most important problems and the main objective of the problem is to optimize a chosen objective function through optimal adjustments of power system control variables while at the same time satisfying system operating conditions with power flow equations and inequality constraints. The equality constraints are the nodal power balance equations, while the inequality constraints are the limits of all control or state variables. The control variables involve the tap ratios of transformers, the generator real power, the generator bus voltages and reactive power of sources. In general, the OPF problem is a large-scale, highly constrained, nonlinear and non-convex optimization problem.

H.W. Dommel and W.F.Tinney [1] firstly presented the solution of optimal power flow. In the past, conventional methods such as interior point method, linear programming and nonlinear programming have been discussed by K. Deb [2] for optimizing engineering problems. The disadvantage of these techniques is that it is not possible to use as an efficient tool in practical systems because of nonlinearity of the problem. Recently many population-based methods have been proposed for solving the OPF problem successfully. Examples of such methods are genetic algorithm (GA), particle swarm optimization (PSO), differential evolution (DE), simulated annealing (SA), Intelligent search evolutionary algorithm
(ISEA) etc. These techniques have been increasingly applied for solving power system optimization problems such as economic dispatch, optimal reactive power flow and OPF for decades.

R.Gnanadass et al. [3] discuss the evolutionary programming algorithm to solve the OPF problem with non-smooth fuel cost function. M.R.Al Rashidi and M.E.EI.Hawary [4] have reported a hybrid particle swarm optimization algorithm to solve the discrete OPF problem with valve loading effect. M. Varadarajan and K.S Swarup [5] proposed differential evolution approach to solve OPF problem with multiple objectives. A.V.Naresh Babu and S.Sivanagaraju [6] proposed a new approach based on two step initialization to solve the OPF problem.

All search intelligence techniques are population based and stochastic in nature and are applied to obtain quality solutions [7] to optimization problems. big-bang and big-crunch (BBBC) developed by Erol and Eksin [8] from the concept of universal evolution, fire fly optimization (FFO) also a heuristic algorithm developed by Dr. Xin-she yang [9] and cuckoo optimization algorithm [10] proposed by X.S. Yang are also a population based search technique used to solve OPF problem.

In this proposed work, optimal power flow problem is handled by the recently introduced bio inspired fruit fly algorithm [11]. This simple and efficient algorithm is tested on the standard IEEE-30 bus system.

\section{PROBLEM FORMULATION}

The main objective this work is to minimize the total production cost of real power for reasons of economics. This can be mathematically written as follows [12].

$\operatorname{Minf}(x, u)$

s.t.

$g(x, u)=$

$h(x, u) \leq 0$

$u \in \mathrm{U}$

where $x=\left[\delta^{T}, V_{L}^{T}\right]^{T}$

$x$ is the state vector of the system with bus voltage angles $\delta$ and load bus voltages $\mathrm{V}_{\mathrm{L}}$. Control variables to optimize the equation 1 are real power generation of generator $\left(P_{g}\right)$, 
terminal voltages of generators $\left(V_{g}\right)$, tap-setting of transformers $\left(t_{\text {tap }}\right)$ and switchable shunts $\left(Q_{s h}\right)$.

$u=\left[P_{g}^{T}, V_{g}^{T}, t_{t a p}^{T}, Q_{s h}^{T}\right]$

Equation (1) is considered as sum of quadratic cost functions of thermal generating units with usual $a_{i}, b_{i}, c_{i}$ cost coefficients.

$F_{T}\left(P_{g}\right)=\sum_{i=1}^{N g} a_{i}+b_{i} P_{g i}+c_{i} P_{g i}^{2} \quad \$ / h r$

This objective is subjected to the following constraints.

\subsection{Equality constraints}

(i) Active power balance at all the buses

$$
\begin{gathered}
P_{i}-P_{g i}+P_{d i}=0 \\
\quad(i=1,2,3, \ldots \ldots \ldots
\end{gathered}
$$

(ii) Reactive power balance at all the nodes

$$
\begin{gathered}
Q_{i}-Q_{g i}+Q_{d i}=0 \\
(i=N G+1, \ldots
\end{gathered}
$$

\subsection{Inequality constraints}

(i) Limits on active power generation

$$
\begin{array}{r}
P_{g i}^{\min } \leq P_{g i} \leq P_{g i}^{\max } \\
(i=1,2, \ldots
\end{array}
$$

(ii) Limits on reactive power generation

$$
\begin{aligned}
& V_{g i}^{\min } \leq V_{g i} \leq V_{g i}^{\max } \\
&(i=1,2, \ldots
\end{aligned}
$$

(iii) Limits on switchable shunt compensators

$$
\begin{aligned}
Q_{s h i}^{\min } \leq & Q_{s h i} \leq Q_{s h i}^{\max } \\
& \left(i=1, \ldots \ldots \ldots \ldots N_{S H}\right)
\end{aligned}
$$

(iv) Limits on tap setting of transformers

$$
\begin{aligned}
t_{\text {tap }}^{\min } \leq & t_{\text {tap }} \leq t_{\text {tap }}^{\max } \\
& \left(i=1, \ldots \ldots \ldots \ldots N_{T}\right)
\end{aligned}
$$

The limits on the control variables of real power generations, voltage magnitudes of generators, transformer tap settings and switchable shunt devices are implicitly handled while generating the parameters randomly. Due to inclusion of penalty terms, equation (6) transforms to a pseudo objective function $(\mathrm{F})$

$$
\begin{aligned}
\min F=F_{T}\left(P_{g}\right)+ & P_{s} \\
& +\sum_{i=1}^{N P Q} P_{V i} \\
& +\sum_{i=1}^{N G} P_{Q i}+\sum_{i=1}^{N L} P_{L i}
\end{aligned}
$$

Here $P_{S}, P_{V i}, P_{Q i}, P_{L i}$ are penalty terms for slack bus generator MW limit violation, Load bus voltage limit violations, generator reactive power limit violations and violations for thermal limits of lies respectively.

\section{FRUIT FLY OPTIMIZATION ALGORITHM}

Fruit Fly Optimization Algorithm is put forward by Taiwanese scholar Pan [11]. It is a new optimization method based on fruit fly's foraging behaviors and most researchers used this algorithm for many optimization problems. Fruit flies are superior to other species in terms of visual senses. They can successfully pick up various odors floating in the air with their olfactory organ, some can even smell food sources 40 kilometers away. Then, they would fly to the food. They may also spot with their sharp vision food or a place where their companions gather.

Fruit fly's foraging characteristics have been summarized and programmed into the following steps, which are:

1: Randomly generate initial position for the swarm of fruit fly.

$$
\text { Init } \mathrm{X} \text { axis; Init } \mathrm{Y} \text { axis }
$$

2: Randomly assign each fruit fly a direction and distance for their movement to look for food with their olfactory organ.

$$
\begin{aligned}
& X_{i}=X_{\text {axis }}+\text { RandomValue } \\
& Y_{i}=Y_{\text {axis }}+\text { RandomValue }
\end{aligned}
$$

Since food position is unknown, the distance (Dist ${ }_{i}$ ) to the origin is estimated first, and the judged value of smell concentration $\left(\mathrm{S}_{i}\right)$, which is the inverse of distance, is then calculated.

$$
\text { Dist }_{i}=\sqrt{\left(X_{i}^{2}+Y_{i}^{2}\right)} ; \quad S_{i}=1 / \text { Dist }_{i}
$$

3: Substitute the judged values of smell concentration $\left(\mathrm{S}_{i}\right)$ into the smell concentration judge function (also called fitness function) to get the smell concentrations $\left(\right.$ Smell $\left._{i}\right)$ of at positions of each and every fruit flies.

$$
\text { Smell }_{\mathrm{i}}=\operatorname{Function}\left(\mathrm{S}_{\mathrm{i}}\right)
$$

4: Identify the fruit fly whose position has the best smell concentration (maximum value)

$$
\text { [bestSmellbestIndex }]=\max (\text { Smell })
$$

5: Keep the best smell concentration value and $x, y$ coordinate; the fruit fly swarm will see the place and fly towards the position.

$$
\begin{aligned}
& \text { Smellbest }=\text { bestSmell } \\
& \qquad \begin{array}{r}
\mathrm{X} \text { axis }=\mathrm{X}(\text { bestIndex })(21) \\
\mathrm{Y} \text { axis }=\mathrm{Y}(\text { bestIndex })(22)
\end{array}
\end{aligned}
$$

6: Enter iterative optimization, repeat steps $2-5$ and judge whether the smell concentration is higher than that in the previous iteration; if so carry out step 6 .

\section{IMPLEMENTATION OF FOA ALGORITHM}

Form an initial generation of NP flies in a random manner respecting the limits of search space. Each fly is a vector of all control variables, i.e. $\left[\mathrm{P}_{\mathrm{g}}, \mathrm{V}_{\mathrm{g}}, \mathrm{T}_{\text {tap }}, \mathrm{Q}_{\mathrm{sh}}\right]$. There are $5 \mathrm{P}_{\mathrm{g}}$ 's, 6 $\mathrm{V}_{\mathrm{g}}$ 's, $9 \mathrm{Q}_{\text {sh }}$ 's and $4 \mathrm{~T}_{\text {tap }}$ 's in the IEEE-30 system and hence a fly is a vector of size $1 \times 15$. Calculate the smell concentration function values of all flies solution by running the NR load 
flow. The control variable values taken by different flies are incorporated in the system data and load flow is run. The total line loss corresponding to different flies are calculated. The steps followed in this algorithm is given below.

Step 1: Form an initial generation of NP flies in a random manner respecting the limits of search space. Each fruit fly is a vector of all control variables, i.e. $\left[\mathrm{P}_{\mathrm{g}}, \mathrm{V}_{\mathrm{g}}, \mathrm{T}_{\text {tap }}, \mathrm{Q}_{\mathrm{sh}}\right]$. There are $5 \mathrm{P}_{\mathrm{g}}$ 's, $6 \mathrm{~V}_{\mathrm{g}}$ 's, $2 \mathrm{Q}_{\text {sh }}$ 's and $4 \mathrm{~T}_{\text {tap }}$ 's in the IEEE-30 system and hence a fly is a vector of size $1 \times 17$.

Step 2: Calculate the smell concentration values of all flies solution by running the NR load flow. The control variable values taken by different flies are incorporated in the system data and load flow is run. The total line loss corresponding to different flies are calculated.

Step 3: Determine the best fly which has global best smell concentration using equation (19). The flies are arranged in the ascending order their (smell concentration) and the first fly will be the candidate with best smell (minimum cost).

Step 4: Generate new fly around the global best fly by adding/subtracting a normal random number according to equation (20). It should be ensured that the control variables are within their limits otherwise adjust the values.

Step 5: Repeat steps 2-5 until best smell concentration is not achieved.

\section{NUMERICAL RESULTS AND DISCUSSIONS}

The effectiveness of the proposed optimization method is tested on the standard IEEE-30 bus system. The necessary line and bus data of the system are taken from [13]. The dimension of this problem is 12 including 6 generator voltages, 4 transformers tap settings and 2 static var compensators. The first bus is the slack bus and its real power generation is not controlled for OPF. System total load considered is the base load (2.834 p.u.+j1.2620 p.u.) on 100 MVA base.

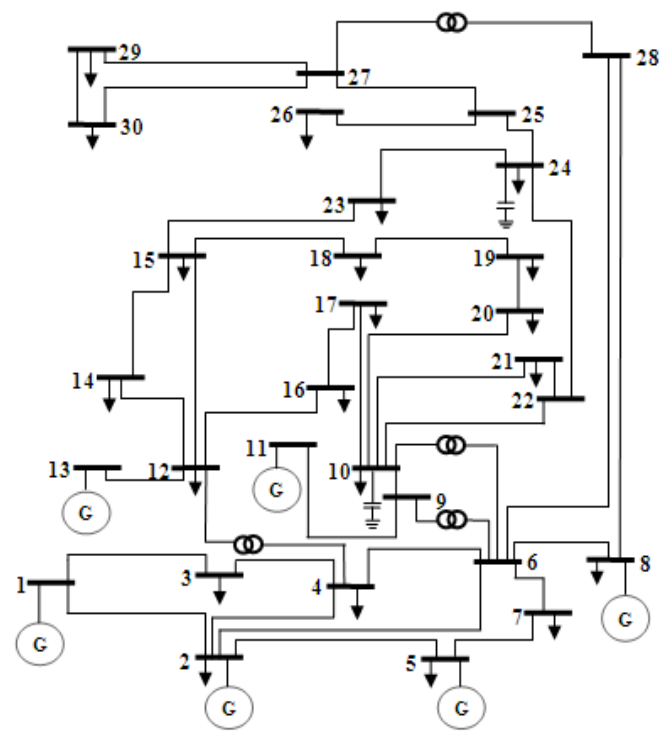

Figure 1. One line diagram of IEEE 30 bus system

Quadratic cost function is used for calculating the total fuel cost. The real power generation limits and three cost coefficients are given in table 1.

Table 1. Real Power Limits and Cost Coefficients of Generators

\begin{tabular}{|l|l|l|l|l|l|}
\hline \multirow{2}{*}{$\begin{array}{l}\text { Bus } \\
\text { No }\end{array}$} & $\begin{array}{l}\text { Real power output } \\
\text { limit(MW) }\end{array}$ & \multicolumn{3}{l|}{ Cost Coefficients } \\
\cline { 2 - 6 } & Min & Max & a & b & c \\
\hline 1 & 50 & 200 & 0 & 2.00 & 0.00375 \\
\hline 2 & 20 & 80 & 0 & 1.75 & 0.01750 \\
\hline 5 & 15 & 50 & 0 & 1.00 & 0.06250 \\
\hline 8 & 10 & 35 & 0 & 3.25 & 0.00834 \\
\hline 11 & 10 & 30 & 0 & 3.00 & 0.02500 \\
\hline 13 & 12 & 40 & 0 & 3.00 & 0.02500 \\
\hline
\end{tabular}

\section{Case1. OPF problem}

In this case, the fuel cost of generators is minimized without taking into account the possible violation of line flow limits. The control parameters are adjusted within their limits and the minimum total fuel cost is obtained. The optimal values of the control variables taken by the proposed algorithm in OPF are given in table 2 .

Table 2. Optimal variables for IEEE 30- bus system

\begin{tabular}{|l|l|l|l|l|}
\hline $\begin{array}{l}\text { Variables } \\
\text { Base }\end{array}$ & $\begin{array}{l}\text { Base } \\
\text { case }\end{array}$ & FFO [14] & $\begin{array}{l}\text { BB- } \\
\text { BC[14] }\end{array}$ & FOA \\
\hline $\operatorname{Pg} 1(\mathrm{pu})$ & 0.9870 & 1.765171 & 1.749672 & 1.764884 \\
\hline $\operatorname{Pg} 2(\mathrm{pu})$ & 0.8 & 0.487865 & 0.481406 & 0.486839 \\
\hline $\operatorname{Pg} 5(\mathrm{pu})$ & 0.5 & 0.214746 & 0.208195 & 0.213967 \\
\hline $\operatorname{Pg} 8(\mathrm{pu})$ & 0.2 & 0.216439 & 0.222772 & 0.193176 \\
\hline $\operatorname{Pg} 11(\mathrm{pu})$ & 0.2 & 0.119801 & 0.141101 & 0.128098 \\
\hline $\operatorname{Pg} 13(\mathrm{pu})$ & 0.2 & 0.120276 & 0.120002 & 0.129209 \\
\hline $\operatorname{Vg} 1(\mathrm{pu})$ & 1.06 & 1.085421 & 1.087797 & 1.1000 \\
\hline $\operatorname{Vg} 2(\mathrm{pu})$ & 1.043 & 1.066785 & 1.065492 & 1.0890 \\
\hline $\operatorname{Vg} 5(\mathrm{pu})$ & 1.013 & 1.034902 & 1.035512 & 1.0649 \\
\hline
\end{tabular}




\begin{tabular}{|l|l|l|l|l|}
\hline $\mathrm{Vg} 8(\mathrm{pu})$ & 1.015 & 1.012432 & 1.032453 & 1.0760 \\
\hline $\mathrm{Vg} 11(\mathrm{pu})$ & 1.082 & 1.069076 & 1.063822 & 1.0528 \\
\hline $\mathrm{Vg} 13(\mathrm{pu})$ & 1.071 & 1.059076 & 1.010111 & 1.0708 \\
\hline $\mathrm{Q} h 10(\mathrm{pu})$ & 0.19 & 0.04 & 0.04 & 0.07 \\
\hline Qsh12(pu) & 0 & 0.03 & 0.01 & 0 \\
\hline Qsh15(pu) & 0 & 0.02 & 0.02 & 0 \\
\hline Qsh17(pu) & 0 & 0.04 & 0.02 & 0 \\
\hline Qsh20(pu) & 0 & 0.04 & 0.02 & 0 \\
\hline Qsh21(pu) & 0 & 0.04 & 0.05 & 0 \\
\hline Qsh23(pu) & 0 & 0.03 & 0.04 & 0 \\
\hline Qsh24(pu) & 0.043 & 0.03 & 0.04 & 0.07 \\
\hline Qsh29(pu) & 0 & 0.02 & 0.02 & 0 \\
\hline t1(6-9) & 0.978 & 0.9850 & 1.0950 & 1.0362 \\
\hline t2(6-10) & 0.969 & 0.9650 & 0.9600 & 1.0643 \\
\hline$t 3(4-12)$ & 0.932 & 0.9900 & 1.0100 & 1.0977 \\
\hline t4(28-27) & 0.968 & 1.005 & 1.015 & 1.0478 \\
\hline $\begin{array}{l}\text { Total } \\
\text { generation } \\
(\mathrm{pu})\end{array}$ & 2.887 & 2.9243 & 2.9231 & 2.83400 \\
\hline Cost(\$/hr) & 900.5211 & 800.6803 & 800.8949 & 799.9787 \\
\hline
\end{tabular}

The efficiency of the algorithm is proved by comparing its performance with that of other recently reported algorithms like EP, IEP and DE. It is obvious from table 3 that the reduction in the total fuel cost is quite encouraging. The cost obtained by other methods is about $802 \mathrm{USD} / \mathrm{hr}$ whereas FOA has achieved $799.9787 \mathrm{USD} / \mathrm{hr}$ and this is a great advantage

Table 3. Total fuel cost obtained by different methods

\begin{tabular}{|l|l|l|l|}
\hline $\begin{array}{l}\text { IEEE-30 } \\
\text { bus } \\
\text { system }\end{array}$ & BB-BC [14] & FFO [14] & FOA \\
\hline Cost & 800.8949 & 800.6803 & 799.9787 \\
\hline
\end{tabular}

Strength of an optimization technique is characterized by its convergence speed or the number of iterations taken to get the optimized results. Figure 2 graphically depicts the excellent convergence quality of FOA algorithm. In this work, the minimum fuel cost is obtained within 140 iterations. This proves the effectiveness of the algorithm.

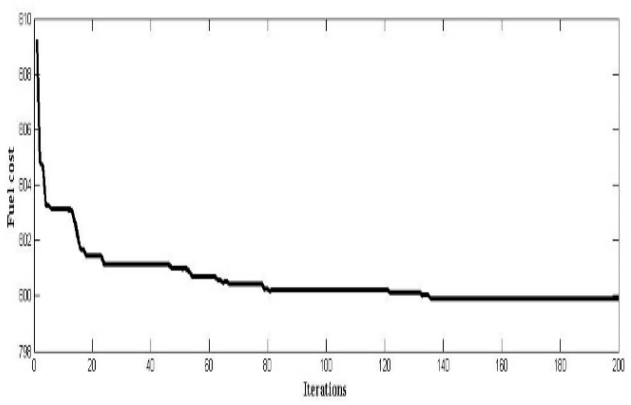

Figure 2. Convergence characteristics of OPF

\section{Case 2. Security constraint OPF problem}

OPF results forces some of the lines in the system to carry power much closer to their limit or violation of limit when only fuel cost is taken as the objective. This will affect the security of the power system. This can be solved by adding line flows as a constraint in the optimization process.

Optimization of fuel cost in the test system taken forces line 1-2 and line 6-8 to carry power beyond their ratings. Power flow through the line 1-2 is 135.61 MVA against its capacity of 130 MVA showing violation. 44.75 MVA power flows in line 6-8 while its rating is $32 \mathrm{MVA}$. Though the cost obtained is minimum, the security of the system is under threat.

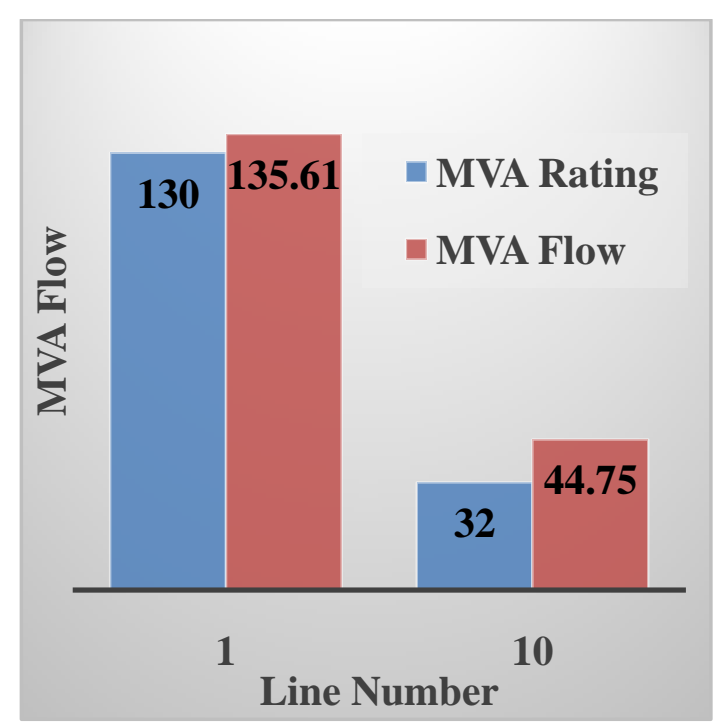

Figure 3. Power flow violation

This case addresses the above stated problem by adding the line flows as a constraint. The optimal values of control parameter corresponding to the best results are tabulated in table 4 . The fuel cost is slightly higher but acceptable as security is more important than economy. 
Table 4. Optimal variables for IEEE 30- bus system

\begin{tabular}{|l|l|l|}
\hline Variables Base & OPF & SCOPF \\
\hline Pg1(pu) & 1.76488 & 1.76304 \\
\hline Pg2(pu) & 0.486839 & 0.491673 \\
\hline Pg5(pu) & 0.213967 & 0.243553 \\
\hline Pg8(pu) & 0.193176 & 0.330963 \\
\hline Pg11(pu) & 0.128098 & 0.187659 \\
\hline Pg13(pu) & 0.129209 & 0.181579 \\
\hline Vg1(pu) & 1.1000 & 1.10000 \\
\hline Vg2(pu) & 1.0890 & 1.0874 \\
\hline Vg5(pu) & 1.0649 & 1.0672 \\
\hline Vg8(pu) & 1.0760 & 1.0701 \\
\hline Vg11(pu) & 1.0528 & 1.0767 \\
\hline Vg13(pu) & 1.0708 & 1.0670 \\
\hline Qsh10(pu) & 0.07 & 2.0000 \\
\hline Qsh12(pu) & 0 & 0 \\
\hline Qsh15(pu) & 0 & 0 \\
\hline Qsh17(pu) & 0 & 0 \\
\hline Qsh20(pu) & 0 & 0 \\
\hline Qsh21(pu) & 0 & 0 \\
\hline Qsh23(pu) & 0 & 0 \\
\hline Qsh24(pu) & 0.07 & 17.0000 \\
\hline Qsh29(pu) & 0 & 0 \\
\hline t1(6-9) & 1.0362 & 1.0490 \\
\hline 22(6-10) & 1.0643 & 1.0078 \\
\hline t3(4-12) & 1.0977 & 1.0192 \\
\hline 44(28-27) & 1.0478 & 1.0235 \\
\hline Total Real power & 2.83400 & 2.834 \\
\hline Cost( $(\mathrm{hr})$ & 799.9787 & 808.2497 \\
\hline
\end{tabular}

After optimization, the line flow violations are removed. Figure 4 compares the line rating and power flow through the line in post optimization period. It is clear from the figure that power flow in the violated lines are relieved considerably. In this case, the optimization ensures both fuel cost minimization and security of the power system.

The proposed algorithm performs better in both OPF and SC$\mathrm{OPF}$ in minimizing the fuel cost. Optimization of fuel cost also includes the violation of power flow through the lines.

Since the algorithm behaves in a better way in handling the constraint of power flow limit, this algorithm outperforms the other algorithms in the literatures.

The algorithm handles well both constrained and unconstrained optimization problems. Here, case 1 is an unconstraint case and case 2 a constrained one. The behaviour of the algorithm in these cases proves its ability to optimize different optimization problems.

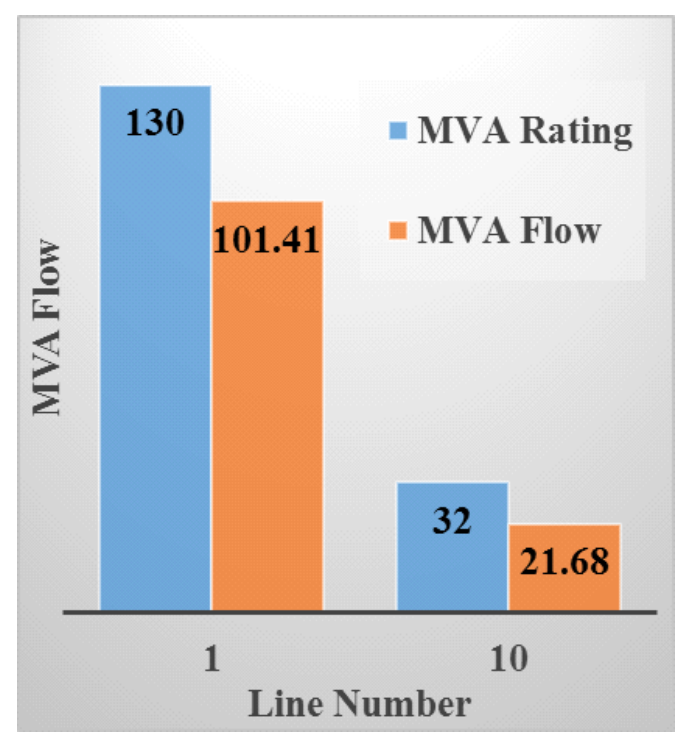

Figure 4. Power flow violation 
Convergence efficiency of FOA is good in this case also. It is clear from figure 5 that convergence occur at about $120^{\text {th }}$ iteration. This case a constrained optimization but still the algorithm converges to the best results. The reliability of the algorithm is proved that it can be used for any optimization algorithm.

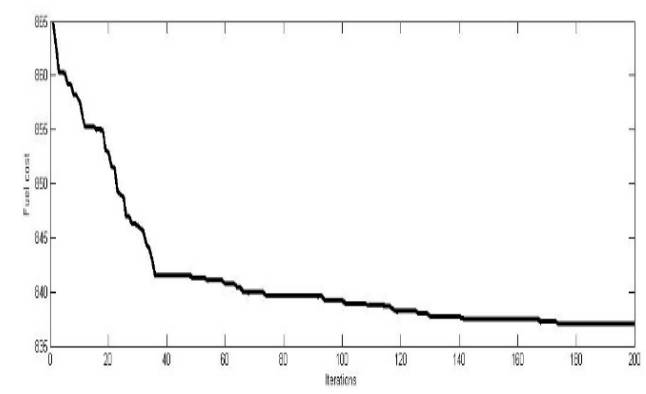

Figure 5. Convergence characteristics of SC-OPF

\section{CONCLUSIONS}

FOA is new and bio inspired optimization algorithm mimicking the food searching behavior of fruit flies. Because of less number of operators and parameters, the algorithm is found to be simple in implementation. The results obtained by the proposed algorithm on IEEE-30 bus system is compared with the other algorithm in the literatures. It is obvious from the test results that FOA outperforms the other recently introduced optimization techniques in optimal power flow problem. The algorithm achieves the results in relatively less number of iterations. Speed of convergence of the algorithm is also examined to establish the strength of the algorithm. Therefore, it is believed that this algorithm may be exploited for other power system operations like economic load dispatch, optimal power flow, voltage stability improvement etc.

\section{REFERENCES}

[1] Dommel, Hermann W., and William F. Tinney. "Optimal power flow solutions." IEEE Transactions on power apparatus and systems 10 (1968): 1866-1876.

[2] Deb, Kalyanmoy. Optimization for engineering design: Algorithms and examples. PHI Learning Pvt. Ltd., 2012.

[3] Venkatesh, P., R. Gnanadass, and Narayana Prasad Padhy. "Comparison and application of evolutionary programming techniques to combined economic emission dispatch with line flow constraints." IEEE Transactions on Power systems 18.2 (2003): 688-697.
[4] AlRashidi, M. R., and M. E. El-Hawary. "Hybrid particle swarm optimization approach for solving the discrete OPF problem considering the valve loading effects." IEEE transactions on power systems 22.4 (2007): 2030-2038.

[5] Varadarajan, M., and K. Shanty Swarup. "Solving multiobjective optimal power flow using differential evolution." IET Generation, Transmission \& Distribution 2.5 (2008): 720-730.

[6] Babu, AV Naresh, and Sirigiri Sivanagaraju. "Optimal power flow with FACTS device using two step initialization based algorithm for security enhancement considering credible contingencies." Advances in Power Conversion and Energy Technologies (APCET), 2012 International Conference on. IEEE, 2012.

[7] Abido, M. A. "Optimal power flow using particle swarm optimization." International Journal of Electrical Power \& Energy Systems 24.7 (2002): 563-571.

[8] Erol, Osman K., and Ibrahim Eksin. "A new optimization method: big bang-big crunch." Advances in Engineering Software 37.2 (2006): 106-111.

[9] Yang, Xin-She. "Multiobjective firefly algorithm for continuous optimization." Engineering with Computers 29.2 (2013): 175-184.

[10] Yang, Xin-She, and Suash Deb. "Cuckoo search via Lévy flights." Nature \& Biologically Inspired Computing, 2009. NaBIC 2009. World Congress on. IEEE, 2009.

[11] Pan, Wen-Tsao. "A new fruit fly optimization algorithm: taking the financial distress model as an example." Knowledge-Based Systems 26 (2012): 69-74.

[12] Abido, M. A. "Optimal power flow using tabu search algorithm." Electric Power Components and Systems 30.5 (2002): 469-483.

[13] Ghasemi, Mojtaba, et al. "An improved teachinglearning-based optimization algorithm using Lévy mutation strategy for non-smooth optimal power flow." International Journal of Electrical Power \& Energy Systems 65 (2015): 375-384.

[14] Rao, CV Gopala Krishna, and G. Yesuratnam. "Big-Bang and Big-Crunch (BB-BC) and FireFly Optimization (FFO): Application and Comparison to Optimal Power flow with Continuous and Discrete Control Variables." International Journal on Electrical Engineering and Informatics 4.4 (2012): 575. 